Proceedings of the 2011 Winter Simulation Conference

S. Jain, R. R. Creasey, J. Himmelspach, K. P. White, and M. Fu, eds.

\title{
RETHINKING THE INITIALIZATION BIAS PROBLEM IN STEADY-STATE DISCRETE EVENT SIMULATION
}

\author{
Winfried Grassmann \\ Department of Computer Science \\ University of Saskatchewan \\ 110 Science Court \\ Saskatoon, SK S7N 5C9
}

\begin{abstract}
The state in which a discrete event simulation is started causes the estimators for equilibrium measures obtained from the simulation to be biased, and to reduce this bias, the collection of data is delayed until a so-called warm-up period is completed. In this paper, we determine the optimal warm-up periods for steady-state discrete event simulations. We do this by using deterministic numerical methods, that is, methods not using random numbers. We found that in the systems investigated, transient expectations give no indication regarding the optimal length of the warm-up periods, which is counterintuitive. This requires some re-evaluation of some of commonly held opinions about the factors one should take into account when determining warm-up periods. Such factors will also be discussed.
\end{abstract}

\section{INTRODUCTION}

The initial bias problem arising in discrete event simulation has been discussed in numerous articles (see (Awad 2006), (Mahadjan and Ingalls 2004), (Robinson 2007), (White 1997), and the review paper (Pawlikowski 1990), and references in these papers). It is described in most textbooks dealing with simulation (see e.g. (Asmussen and Glynn 2007), (Banks, Carson, Nelson, and Nicol 2005), (Law and Kelton 2000)). Yet, some results given in literature may need modification and/or elaboration. One reference, which we found very valuable, is in the book by (Tocher 1963, page 176), who uses the warm-up period to find what he calls "typical conditions".

First, we describe the problem: Let $R(t)$ be the process to be simulated as part of a discrete event simulation, and assume that $\mathrm{E}(R(t))$ converges toward an equilibrium value $\mathrm{E}(R)$, where $\mathrm{E}(R)$ is independent of the starting state. To estimate $\mathrm{E}(R)$ by simulation, we use a time average. In many simulations, this time average is only taken after a warm-up period of length $\tau \geq 0$, that is, the time average becomes $\bar{R}(T)=\frac{1}{T-\tau} \int_{\tau}^{T} R(t) d t$. Under very general conditions, $\bar{R}(T)$ converges with probability 1 toward $\mathrm{E}(R)$. Of course, we would like to select an initial state $i$ and a value of $\tau$ such that the difference between $\bar{R}(T)$ and $\mathrm{E}(R)$ is small, while we require that $T$ is less than or equal to some constant. Specifically, we use the mean squared error $\operatorname{MSE}_{i}(\bar{R}(T))=\mathrm{E}\left((\bar{R}(T)-\mathrm{E}(R))^{2}\right)$ to judge the quality of our estimate.

Suppose, first, that the initial state $i$ is given. In this case, one would expect that the transient behavior of the system would shed some light to what might be a good value for $\tau$, the length of the warm-up period. We show, by using examples, that this is not necessarily the case.

In contrast to other efforts, we do not use random numbers to obtain our results, but we use non-Monte Carlo methods, also called deterministic methods. Specifically we use a modification of a method described in (Grassmann 1987) to find the mean squared error in question. Also, asymptotic results are available ( (Grassmann 2008), (Grassmann and Luo 2005), (Hazen and Pritsker 1980), (Kelton and Law 1983), (Kelton and Law 1985), (Madansky 1976), (van Moorsel, Kant, and Sanders 1996), (Whitt 1992), (Wilson and Prisker 1978a), (Wilson and Prisker 1978b), and references in these papers). We also should mention 


\section{Grassmann}

the work of (Reynolds 1975) and references therein. We note that all these methods are restricted to continuous-time Markov chains. However, our models are only used as examples to clarify the issue, and to show that commonly held opinions can be wrong. At the very minimum, this should make the reader aware of potential problems which can then be further investigated in more complex models.

\section{TRANSIENT SOLUTIONS AND WARM-UP PERIODS}

Several papers seem to suggest that the transient expectations can be used as a guide for determining the optimal value of the warm-up period. Let us see how this works out in concrete examples. As our first model, consider the $\mathrm{M} / \mathrm{M} / 1 / 10$ model, that is, a queuing system with Poisson arrivals, exponential service times, one server, and a maximum of 10 elements in the system. The problem is to estimate by using simulation the expected number in the system. For this purpose, let $R(t)$ represent the number in the system, which means that $\mathrm{E}(R)$ is to be found. We want to determine a good value for the length of the warm-up period $\tau$, and we ask if the transient solution $\mathrm{E}(R(t))$ is helpful in this regard. Hence, consider Figure 1, which gives the results for $\mathrm{E}(R(t))$ when the arrival rate is $\lambda=0.8$ and the service rate is $\mu=1$. One can

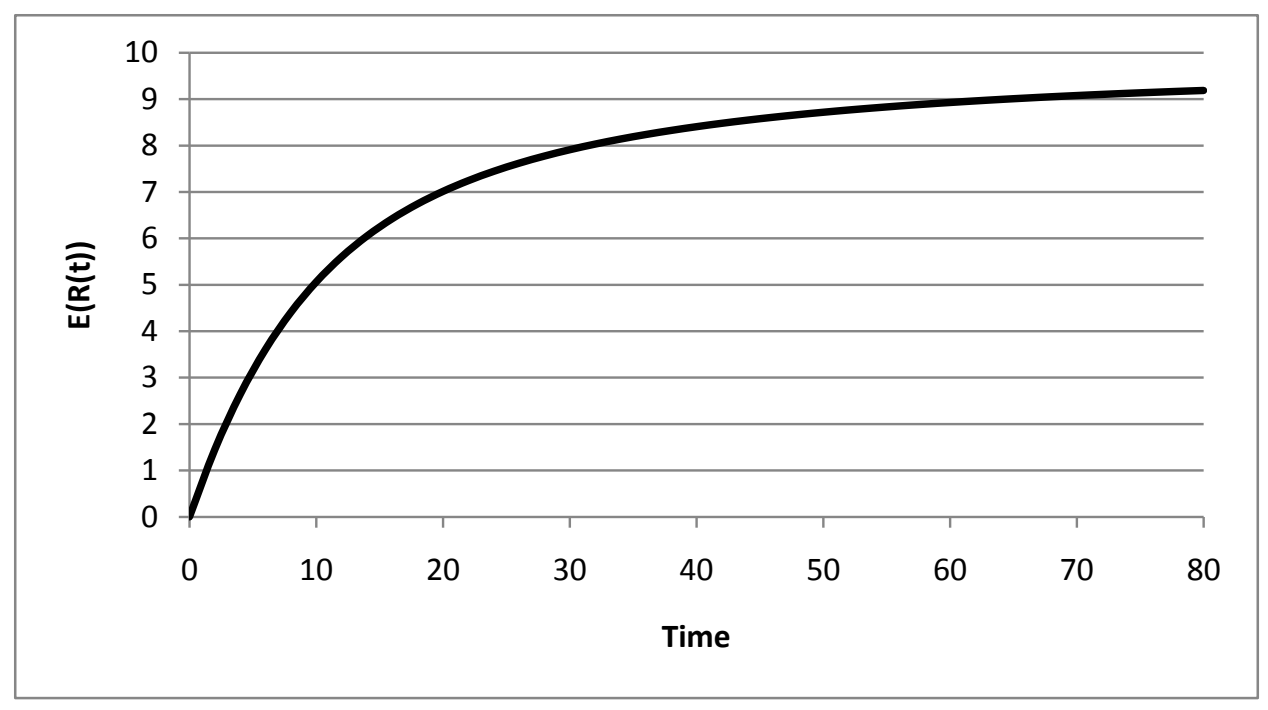

Figure 1: Expected number in M/M/1/10 system with $\lambda=0.8, \mu=1$.

see that at $t=23.6, \mathrm{E}(R(t))$ reaches $90 \%$ and at $t=31.9$ it reaches $95 \%$ of its equilibrium expectation $\mathrm{E}(R)$. Hence, one would expect that a warm-up period $\tau$ around 25 should do. To see how this works out, we calculated the mean squared error for $\tau$ ranging from 0 to 25 given $T=1000$. The result: the mean squared error is lowest when $\tau=0$, where it has a value of 0.171 . The MSE is increasing with $\tau$, reaching 0.177 when $\tau=25$. Hence, instead of using a warm-up period of 25, one should use no warm-up period! In other words, the transient solution given in Figure 1 is misleading.

One may object that the $\mathrm{M} / \mathrm{M} / 1 / 10$ queue is rather special, and that the finding above is an exception. However, in all cases we looked at, we found that transient solutions greatly overestimate the warm-up period. The next two tables show this. They give the results for the $\mathrm{M} / \mathrm{M} / 10 / 30$ and a system of tandem queues. In both cases, the objective is to determine the expectation of the total number in the system. The $\mathrm{M} / \mathrm{M} / 10 / 30$ queue has an arrival rate of $\lambda=8$ and a service rate of $\mu=1$. The results are given in Table 1, which compares the values where $\mathrm{E}(R(t))$ reaches $90 \%$ and $95 \%$ of their equilibrium values with the optimal value for $\tau$. Table 2 gives the numbers for a tandem queue with blocking and the number of centers ranging from 2 to 4 . All arrivals have to go through all the centers in sequence. Also, arrivals are exponential, with arrival rates as given in the table. The the service times are exponential with a rate of 1. The number in each center is limited to 4 . From these tables, one notes that the optimal values for 


\section{Grassmann}

Table 1: Transient solutions versus optimal warm-up periods M/M/10/30 Queue.

$\begin{array}{rrrr}\lambda & \begin{array}{r}90 \% \\ \text { of equil }\end{array} & \begin{array}{r}95 \% \\ \text { of equil }\end{array} & \tau \text { minimizing } \\ 0.8 & 43.3 & 68.9 & \text { MSE } \\ 0.9 & 85.4 & 124.4 & 1.8 \\ & & \end{array}$

Table 2: Transient solutions and warm-up periods in tandem queues.

$\begin{array}{rrrrr}\lambda & \text { centers } & \begin{array}{r}90 \% \\ \text { of equil }\end{array} & \begin{array}{r}95 \% \\ \text { of equil }\end{array} & \tau \text { minimizing } \\ 0.8 & 2 & 10.9 & 14.4 & 0.6 \\ 0.9 & 2 & 10.6 & 13.9 & 1.4 \\ 0.8 & 3 & 16.0 & 21.5 & 2.5 \\ 0.9 & 3 & 15.6 & 20.8 & 3.8 \\ 0.8 & 4 & 21.6 & 29.3 & 4.9 \\ 0.9 & 4 & 21.0 & 28.2 & 6.6\end{array}$

$\tau$ are, in all cases, only a fraction of the one suggested by the transient solutions. We conclude that in many cases, using transient solutions is misleading. Hence, if we see a title like "The Transient behavior of the M/M/c Queue, with Implication for Steady-State Simulation" as in (Kelton and Law 1985), we have to conclude that this is misleading since there may be no connection between the transient behavior and warm-up periods, contradicting thus the authors of (Kelton and Law 1985). We have to confess, though, that before doing our investigation, we had, like others (Asmussen and Glynn 2007) (Asmussen, Glynn, and Thorisson 1992), the same opinion. Hence, we were in good company. Since our findings are somewhat paradoxical, it is imperative that the initialization bias problem be reexamined, which we do below. First, however, we have to review some basic relations.

\section{IMPORTANT DEFINITIONS AND RELATIONS}

The examples above are all Markovian, in some cases with several state variables, and we will restrict our attention to Markov chains. This is not a major restriction since, as pointed out in (Asmussen, Glynn, and Thorisson 1992) and (Grassmann 2008), most systems can be approximated arbitrary closely by Markov chains. Besides, it would be very difficult to argue that the paradoxical behavior discussed above is restricted to Markov chains. We further assume that the number of states is countable. The state the system at time $t$ will be denoted by $X(t)$. Typically, we are not interested in $X(t)$ per se, but in $R(t)$, which is a function of $X(t)$. For instance, in the tandem queues with $d$ centers discussed above, $X(t)$ would be a vector of dimension $d$, but we were interested in the number in total, that is, the number of all centers added together. This number is our $R(t)$. Since $R(t)$ depends on $X(t)$, we can define $r_{i}$ as the value of $R(t)$ if $X(t)=i$. Note that even though $X(t)$ is Markovian, $R(t)$ typically is not because it does not contain the full information captured by $X(t)$.

We now define

$$
\begin{aligned}
p_{i j}(t) & =\mathrm{P}\{X(t)=j \mid X(0)=i\} \\
\mathrm{E}_{i, \tau}(\bar{R}(T)) & =\mathrm{E}(\bar{R}(T) \mid X(0)=i, \tau) \\
\operatorname{Bias}_{i, \tau}(\bar{R}(T)) & =\mathrm{E}_{i, \tau}(\bar{R}(T))-E(R) \\
\operatorname{Var}_{i, \tau}(\bar{R}(T)) & =\operatorname{Var}(\bar{R}(T) \mid X(0)=i, \tau) \\
\operatorname{MSE}_{i, \tau}(\bar{R}(T)) & \left.=\mathrm{E}\left((\bar{R}(T)-\mathrm{E}(R))^{2} \mid X(0)=i, \tau\right)\right) .
\end{aligned}
$$




\section{Grassmann}

For convenience, we will write $\mathrm{E}_{i}(\bar{R}(T))$ instead of $\mathrm{E}_{i, \tau}(\bar{R}(T))$ when the value of $\tau$ is clear from the context, and $\operatorname{Var}_{i}(\bar{R}(T))$ and $\operatorname{MSE}_{i}(\bar{R}(T))$ must be understood in a similar fashion. It is easy to verify that

$$
\operatorname{MSE}_{i}(\bar{R}(T))=\operatorname{Var}_{i}(\bar{R}(T))+\operatorname{Bias}_{i}^{2}(\bar{R}(T))
$$

Efficient ways to calculate $\mathrm{E}_{i}(\bar{R}(T))$ and $\operatorname{Var}_{i}(\bar{R}(T))$ are given in (Grassmann 1987), and we used these methods to obtain the results presented above.

\section{ASYMPTOTIC RESULTS}

The issue in question becomes clearer if we consider asymptotic results. For large $T$, and $\tau<<T$, we can use the following relations

$$
\begin{aligned}
\operatorname{Bias}_{i, \tau}(\bar{R}(T)) & =B_{i}(\tau) /(T-\tau)+o(1 /(T-\tau)) \\
\operatorname{Var}_{i, \tau}(\bar{R}(T)) & =V /(T-\tau)+o(1 /(T-\tau)) .
\end{aligned}
$$

These relations are discussed in (Asmussen and Glynn 2007), but they appeared earlier in (Grassmann 1982), where we also give a method to find $B_{i, 0}$ and $V$ numerically. For improved methods to find $B_{i, 0}$ and $V$, see (Grassmann 2008) and references therein. Essentially, these methods involve solving a system of linear equations, which are identical to the equilibrium equations, except that the right-hand side is not 0 . Another method to find $B_{i, 0}$ and $V$ is given in (van Moorsel, Kant, and Sanders 1996).

The algorithms mentioned allow one to find $B_{i, \tau}$ for $\tau=0$. To calculate $B_{i, \tau}$ for $\tau>0$, we proceed as follows. The algorithms allow one to choose the initial state $X(0)$ randomly, that is, we can set $\mathrm{P}\{X(0)=j\}=p_{i j}(\tau)$. If the value obtained this way is $B_{i}^{\#}(\bar{R}(T))$, then we can use $B_{i, \tau}(\bar{R}(T))=B_{i}^{\#}(\bar{R}(T-\tau))$.

We now substitute (2) and (3) into (1) to find

$$
\operatorname{MSE}_{i, \tau}(\bar{R}(T))=V /(T-\tau)+B_{i, \tau}^{2} /(T-\tau)^{2}+o(1 /(T-\tau)) .
$$

Hence, $B_{i, \tau}(\bar{R}(T))$ becomes negligible for large $T$. As a concrete example, consider the M/M/1/10 queue described above starting with $i=0$ and no warm-up period. According to (Grassmann 2008), the bias in this model is -0.02729 , whereas the variance is 0.17025 , which yields $\operatorname{MSE}_{0}(\bar{R}(1000))=0.17100$, which differs from the variance only in the third decimal digit. We also note that $B_{0}=27.3$ and $V=174.54$, hence $B_{0} / T$ differs from the bias only in the fourth decimal digit, while $V / T$ differs from the $\operatorname{Var}_{0}(\bar{R}(T))$ only in the third digit. Hence, for $T=1000$, the approximation is quite accurate. One might object that $T=1000$ is too high, but if anything, $T$ should be higher in order to obtain a reasonable accuracy. The $95 \%$ confidence interval for $\mathrm{E}(R)$ is $2.966 \pm 0.809$, which is quite wide. For larger $T$, the accuracy of the asymptotic expressions increases.

The fact that the variance dominates implies that in many cases, we only need to find the initial state $i^{*}$ that minimizes the variance $\operatorname{Var}_{i}(\bar{R}(T))$. This has a counterintuitive consequence: if we select the initial state randomly, say we select the $X(0)$ according to the equilibrium probabilities $\pi_{i}$, we get a variance that exceeds $\operatorname{Var}_{i^{*}}(\bar{R}(T))$ because the variation of $X(0)$ has to be added to the variance. Hence, if we start in equilibrium we do not obtain the lowest possible variance, and, as a consequence, we may not obtain the lowest possible mean squared error. In fact, starting with the equilibrium probabilities in the M/M/1/10 queue discussed above, the mean squared error when starting with $X(0)=0$ is $\operatorname{MSE}_{0}(\bar{R}(1000))=0.1710$, whereas starting in equilibrium results in a mean squared error of $\operatorname{MSE}_{\pi}(\bar{R}(T))=0.1725$, which is higher. In (Grassmann 2008), we therefore concluded that starting in equilibrium, and, by extension, using any warm-up period may be detrimental. We have re-evaluated the situation, and concluded that it does not make sense to use a measure that yields a worse value for starting in equilibrium than for starting deterministically in state 0 . We fixed this problem by $\operatorname{replacing} \operatorname{Var}_{i}(\bar{R}(T))$ by $V / T$. We call the resulting measure modified mean squared error, which is defined as:

$$
\operatorname{MSE}_{i, \tau}^{m}(\bar{R}(T))=V /(T-\tau)+\left(\operatorname{Bias}_{i, \tau}(\bar{R}(T))\right)^{2} .
$$




\section{Grassmann}

As mentioned earlier, the bias becomes negligible for large $T$. Hence, if $T$ is large enough, we only need to minimize $V /(T-\tau)$, and since $V$ is constant, this minimum is reached for $\tau=0$. This result is, of course, only true if the term $\left(\operatorname{Bias}_{i, \tau}(\bar{R}(T))\right)^{2}$ can be neglected. We conclude that if $V$ is large when compared to the bias, in which case $T$ must be large in order to obtain reasonable confidence intervals for the estimator, then it is best to have no warm-up. On the other hand, in the case where $V$ is small, short runs may be sufficient, and a value of $\tau>0$ should be chosen. We should also mention that for small $\tau$, the value of the term $V /(T-\tau)$ changes only very little as $\tau$ changes. Hence, even in cases where the bias is small, its effect on the location of the value of $\tau$ that minimizes the modified mean squared error can be significant. On the other hand, if $\mathrm{MSE}^{m}$ as given in (5) changes so little with $\tau$, starting with $\tau=0$ is still convenient.

Let us now look at the situation from another point of view. If the system becomes more deterministic, in the sense that $V$ decreases, the importance of the bias increases, and so does the need for a warm-up period. $V$, in turn, is determined by the variance $\operatorname{Var}(R)$, the variance of $R(t)$ for a given, but large enough $t$, and by the covariance structure. Hence, in systems with high variance of $R$, or with strong correlation, short or no warm-up periods should be used. On the other hand, if the simulation is started in a state far away from $\mathrm{E}(R)$, the bias becomes significant, and warm-up periods are called for. This matches well with the theory of Tocher who uses the warm-up period to find a typical state.

It is also important to understand the difference between deterministic and stochastic systems. A stochastic system is said to be in equilibrium if its distributions do not change over time, whereas a deterministic system is said to be in equilibrium if the state variables themselves do not change over time. In a stochastic system in equilibrium, the state variables still change, which implies that the sample function gives no indication whether or not the system is in equilibrium. In fact, as soon as the state the system is in is known, its distribution becomes degenerate, and we are no longer in equilibrium. To demonstrate this, consider the $\mathrm{M} / \mathrm{M} / 1$ queue. If we know that $R(t)$, the number in the system at time $t$, then it is easy to show that at time $t+h$, with $h$ small, $\mathrm{E}(R(t+h))=E(R(t))+\lambda h-\mu h$ if $R(t)>0$, and $\mathrm{E}(R(t+h))=E(R(t))+\lambda h$ if $R(t)=0$. Both $\lambda h-\mu h$ and $\lambda h$ are different from 0 , which implies that the expectation changes, meaning that the system is not in equilibrium. Of course, if we run a large number of replications in parallel, then in some cases, $R(t)=0$, and in others, $R(t)>0$, and if the proportion of replications with $R(t)=0$ is proportional to the corresponding equilibrium probability $\pi_{0}=1-\lambda / \mu$, then $E(R(t))$ assumes its equilibrium value $E(R)$.

Of course, there are systems which are almost deterministic, meaning that the state variables change only in a relatively narrow range, we call it equilibrium range. If starting the simulation outside its equilibrium range, one needs, of course, a warm-up period, and this warm-up period should end as soon as one enters into the equilibrium range. Since the states in the equilibrium range are what Tocher would call "typical states", this matches well with our earlier discussion. We conclude that the more deterministic a system, the more important the bias, and, for this reason, $\tau$ must be increased.

\section{CONCLUSIONS}

We conclude: if one starts in a state with a high equilibrium probability, one should not use any warm-up period. The prime example for this is the $\mathrm{M} / \mathrm{M} / 1$ queue, where the empty state has the highest equilibrium probability, and this state should be used as the starting state. However, finding such a state may be difficult in complex systems, and even if such a state can be found, it may be inconvenient to use it as starting state. In this case, one should use the warm-up period for finding a typical state, and the length of the warm-up period should be determined in such a way that the likelihood of finding a such a state is sufficiently large. The fact that one should not use a warm-up period when starting in a state with a high equilibrium probability also explains why the transient solutions can be such poor indicators for finding the length of the warm-up period: transient solutions only reflect the bias, and if the process has a large variation, the bias becomes irrelevant. In this case, the issue is to use as much data as possible as long as the data is typical for the process, and this is achieved by reducing the length of the warm-up period. 


\section{Grassmann}

Our investigations also showed that as the run length increases, the effect of the bias decreases, and, as a consequence, the optimal length of the warm-up period decreases.

\section{ACKNOWLEDGMENTS}

This reserch was partially supported by the NSERC grant of the author. I also like to thank the referees for their valuable suggestions.

\section{REFERENCES}

Asmussen, S., and P. W. Glynn. 2007. Stochastic Simulation: Algorithms and Analysis, Volume 57 of Stochastic Modelling and Applied Probability. New York: Springer Verlag.

Asmussen, S., P. W. Glynn, and H. Thorisson. 1992. "Stationarity Detection in the Initial Transient Problem". ACM Transactions on Modelling and Computer Simulation 2 (3): 130-157.

Awad, H. P. 2006, December. "On an Initial Transient Deletion Rule with Rigorous Theoretical Support". In Proceedings of the 2006 Winter Simulation Conference, edited by L. F. Perrone, F. P. Wieland, J. Liu, B. G. Lawson, D. M. Nicol, and R. M. Fujimoto, 186-191. Piscataway, New Jersey: Institute of Electrical and Electronics Engineers, Inc.

Banks, J., J. S. Carson, B. L. Nelson, and D. Nicol. 2005. Discrete Event Simulation. Englewood Cliffs, NJ: Prentice Hall.

Grassmann, W. K. 1982, December. "Initial Bias and Estimation Error in Discrete Event Simulation". In Proceedings of the 1982 Winter Simulation Conference, edited by Y. W. Chao and O. S. Madrigal, 377-384. Piscataway, New Jersey: Institute of Electrical and Electronics Engineers, Inc.

Grassmann, W. K. 1987. "Means and Variances of Time Averages in Markovian Environments". European Journal of Operations Research 31:132-139.

Grassmann, W. K. 2008. "Warm-up Periods in Simulation Can Be Detrimental". Probabilitiy in Engineering and Informational Sciences 22:415-429.

Grassmann, W. K., and J. Luo. 2005. "Simulating Markov-Reward Processes with Rare Events". ACM Transactions on Modelling and Computer Simulation 15 (2): 138-154.

Hazen, G. B., and A. A. B. Pritsker. 1980. "Formulas for the Variance of the Sample Mean in Finite State Markov Processes". Journal of Statist. Comput. Simul 20:25-40.

Kelton, W. D., and A. M. Law. 1983. "A New Approach for Dealing with the Startup Problem in Discrete Event Simulation". Naval Research Logistics Quarterly 30:641-658.

Kelton, W. D., and A. M. Law. 1985. "The Transient Behavior of the M/M/c Queue, with Implication for Steady-State Simulation”. Operations Research 33:378-396.

Law, A. M., and W. D. Kelton. 2000. Simulation Modelling and Analysis. Third ed. New York: McGraw Hill.

Madansky, A. 1976. "Optimal Conditions for a Simulation Problem”. Operations Research 24:572-577.

Mahadjan, P. S., and R. G. Ingalls. 2004, December. "Evaluation of Methods Used to Detect Warm-up Period in Steady State Simulation". In Proceedings of the 2004 Winter Simulation Conference, edited by R. G. Ingalls, M. D. Rossetti, J. S. Smith, and B. A. Peters, 663-671. Piscataway, New Jersey: Institute of Electrical and Electronics Engineers, Inc.

Pawlikowski, K. 1990. "Steady-state simulation of queueing processes: a survey of problems and solutions". ACM Computing Surveys 22 (2): 123-170.

Reynolds, J. F. 1975. "The Covariance Structure of Queues and Related Processes-a Survey of Recent Work". Adv. Appl. Prob. 7:383-415.

Robinson, S. 2007. "A Statistical Process Control Approach to Selecting a Warm-up Period for a Discreteevent Simulation". European Journal of Operationonal Research 176:332-346.

Tocher, K. D. 1963. The Art of Simulation. London: English University Press. 


\section{Grassmann}

van Moorsel, A. P. A., L. A. Kant, and W. H. Sanders. 1996. "Computation of the Asymptotic Bias and Variance for Simulation of Markov Reward Models". In Proceeding of the 29th Simulation Symposium, edited by T. F. Znati and P. A. Wilsey, 173-181. New Orleans, LA: IEEE.

White, K. P. 1997. “A Effective Truncation Heuristic for Bias Reduction in Simulation Output”. Simulation 69 (6): 323-334.

Whitt, W. 1992. "Asymptotic Formulas for Markov Processes with Applications to Simulation". Operations Research 40 (2): 279-291.

Wilson, J. R., and A. A. B. Prisker. 1978a. "Evaluation of Startup Policies in Simulation Experiments". Simulation 31:79-88.

Wilson, J. R., and A. A. B. Prisker. 1978b. "A Survey of Research on the Simulation Startup Problem". Simulation 31:55-58.

\section{AUTHOR BIOGRAPHY}

Winfried Grassmann is Professor Emeritus in the Department of Computer Science, University of Saskatchewan in Saskatoon. Before joining this institution in 1969, he was working in the Operations Research Department of Swissair, the then Swiss flag carrier, where he was one of the creators of the solution Grassmann/Oesch, one of the most successful OR applications of the company. He has written numerous articles in leading journals, mainly on queueing and related areas, but also on simulation. He pioneered the so-called randomization method as a numerical tool, a method that allows one to find transient solutions of continuous-time Markov chains. He also is the inventor of the so-called GTH method, or Grassmann/Taksar/Heyman method. He has written two books, and edited a third one. He is presently area editor of the INFORMS Journal on Computing. For his contributions to Operations Research, he received both the Merit Award and the Service Award of the Candadian Operational Research Society. His email address is grassman@cs.usask.ca. 